\begin{tabular}{|c|l|}
\hline Title & Hydrogen sensing characteristics and mechanism of Pd/A IGaN GaN Schottky diodes subjected to oxygen gettering \\
\hline Author(s) & Hasegawa, Hideki; A kazawa, Masamichi \\
\hline Citation & $\begin{array}{l}\text { Journal of V acuum Science \& Technology B : Microelectronics and Nanometer Structures, 25(4), 1495-1503 } \\
\text { https:/doi.org/10.1116/1.2750343 }\end{array}$ \\
\hline Issue Date & 2007-07 \\
\hline Doc URL & http://hdl.handle.net/2115/30277 \\
\hline Rights & O2007 A merican V acuum Society \\
\hline Type & article \\
\hline File Information & JVSTB25-4.pdf \\
\hline
\end{tabular}

Instructions for use 


\title{
Hydrogen sensing characteristics and mechanism of Pd/AIGaN/GaN Schottky diodes subjected to oxygen gettering
}

\author{
Hideki Hasegawa ${ }^{\text {a) }}$ and Masamichi Akazawa \\ Research Center for Integrated Quantum Electronics (RCIQE), and Graduate School of Information Science \\ and Technology, Hokkaido University, North 13 West 8, Sapporo 060-8628, Japan
}

(Received 14 January 2007; accepted 7 April 2007; published 31 July 2007)

\begin{abstract}
Hydrogen sensing characteristics in vacuum and in air were investigated on Pd Schottky diodes that were formed on AlGaN/GaN two-dimensional electron gas wafer and subjected to a surface control process for oxygen gettering. By applying the surface control process, leakage currents in $\mathrm{Pd} / \mathrm{AlGaN} / \mathrm{GaN}$ Schottky diode were greatly reduced. Such diodes showed high hydrogen detection sensitivities and fast turn-on and -off characteristics in air, although they showed very slow turn-off behavior in vacuum. From detailed measurements of current-voltage $(I-V)$, capacitance-voltage $(C-V)$, and current transient characteristics, the sensing mechanism was explained in terms of Schottky barrier height reduction caused by formation of interface dipole by atomic hydrogen. It was shown that dipole formation is controlled in air by the Langmuir isotherm type adsorption behavior, including the reaction between atomic hydrogen and oxygen. Discrepancies in Schottky barrier height values deduced from $I-V$ and $C-V$ measurements have indicated that current transport is not by the standard thermionic emission process, but by the thermionic field emission process through the thin surface barrier (TSB) in accordance with the TSB model. (C) 2007 American Vacuum Society. [DOI: 10.1116/1.2750343]
\end{abstract}

\section{INTRODUCTION}

Recent trends toward the so-called ubiquitous network era combined with the progress of nanotechnology are rapidly opening up a new horizon for application areas of III-V nanoelectronics, combining information technology, nanotechnology, and biotechnology. Particularly, sensor applications are receiving increased attention in view of the construction of sensor networks ${ }^{1}$ that are formed by integrated sensor chips with wireless communication capabilities. III-V materials such as GaAs, InP, GaN, and their heterostructures are good platforms for such applications, since they are industrially proven materials for constructing high performance communication devices and high speed signal processing integrated circuits. Additionally, their superb transport properties are surface sensitive for sensing physical, chemical, and biochemical information.

Particularly, the AlGaN/GaN system is an attractive material for constructing various chemical and biochemical sensors in addition to their photonic and high-power electronic device applications. This is because of the following unique features: (1) The bulk and surface properties are chemically stable; (2) a high density two-dimensional electron gas (2DEG) is available near surface, which allows highly sensitive detection of surface phenomena; (3) as compared with traditional III-V semiconductors such as GaAs, InP, and related ternary and quaternary materials, the material is environment friendly; (4) due to large band gap energies, the material allows sensing operation at high temperatures; and (5) Realization of wireless sensor chips is feasible by on-chip cointegration with heterostructure field effect transistor

a) Author to whom correspondence should be addressed; electronic mail: hasegawa@rciqe.hokudai.ac.jp
(HFET) circuits for sensor signal processing and wireless communications. One of the demerits of this material system as compared with various known sensor materials is its relatively high cost. However, this demerit may be counterbalanced by the advantage brought by forming integrated wireless sensor chips.

However, surfaces of group III nitrides are still neither well understood nor well controlled up to now. For example, large leakage currents flow in AlGaN/GaN Schottky diodes and Schottky gates of AlGaN/GaN HFETs. They not only deteriorate performances of power rectifiers and transistors, but also sensor performances, as our initial effort on Schottky diode type hydrogen sensors indicated. ${ }^{2}$ Nitridebased hydrogen sensors reported by other workers also seem to suffer from this problem. ${ }^{3-7}$ Recently, we explained the leakage currents in nitride-based Schottky barriers by the thin surface barrier (TSB) model ${ }^{8,9}$ and, based on this, we have successfully reduced leakage currents in Ni/Au/AlGaN Schottky diodes by applying a novel surface control process for oxygen gettering. 10

The purpose of this article is to investigate hydrogen sensing characteristics and mechanism of a $\mathrm{Pd} / \mathrm{AlGaN} / \mathrm{GaN}$ Schottky diode subjected to the oxygen gettering process. After oxygen gettering, the diode showed high hydrogen detection sensitivities and fast turn-on and turn-off characteristics in air. Sensing dynamics and mechanism were explained in terms of Schottky barrier height $(\mathrm{SBH})$ reduction due to the hydrogen-induced interface dipole. Here, dipole formation is controlled by the Langmuir isotherm, including the reaction between atomic hydrogen and oxygen. A large discrepancy was observed between the SBH values by capacitance-voltage measurements and those by current- 


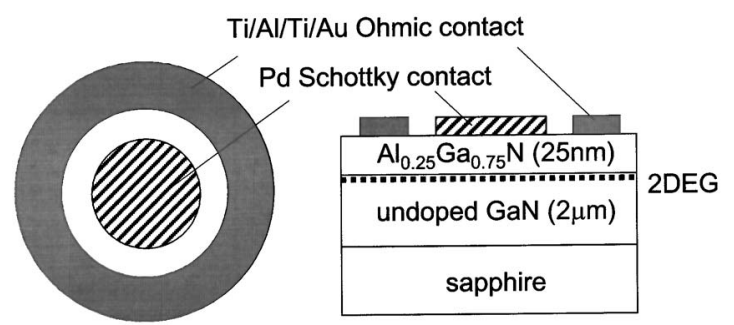

(a)

(1) $\mathrm{N}^{*}$ radical irradiation

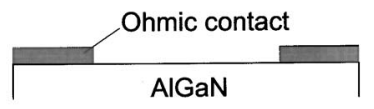

(2) Al (1 nm) deposition by MBD

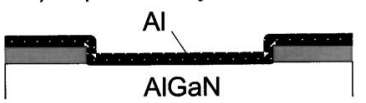

(3) UHV anneal

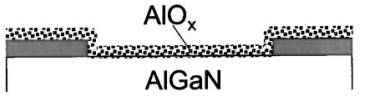

(b)

FIG. 1. (a) Structure of the Pd/AlGaN/GaN diode and (b) the surface control process for oxygen gettering.

voltage measurements. This was explained by the TSB model, where current transport is due to the thermionic field emission through the TSB region.

\section{EXPERIMENT}

The structure of the Schottky diode used in this study for hydrogen sensing is shown in Fig. 1(a). Circular Pd Schottky diodes with $\mathrm{Ti} / \mathrm{Al} / \mathrm{Ti} / \mathrm{Au} \mathrm{Ohmic}$ ring electrodes were formed on an $\mathrm{Al}_{0.25} \mathrm{Ga}_{0.75} \mathrm{~N} / \mathrm{GaN}$ heterostructure wafer grown on sapphire. The wafer had a sheet carrier concentration of $1.1 \times 10^{13} \mathrm{~cm}^{-2}$ and a Hall mobility of $1300 \mathrm{~cm}^{2} / \mathrm{V} \mathrm{s}$ at room temperature (RT). $\mathrm{Ti} / \mathrm{Al} / \mathrm{Ti} / \mathrm{Au}$ Ohmic electrodes were annealed at $800{ }^{\circ} \mathrm{C}$ for $1 \mathrm{~min}$.

In order to remove oxygen from the $\mathrm{AlGaN}$ layer, a surface control process, which we recently proposed, ${ }^{10}$ was applied before Pd deposition. As shown in Fig. 1(b), it consisted of the following steps: (1) Treatment of AlGaN surface in rf-excited plasma assisted nitrogen radicals at $300{ }^{\circ} \mathrm{C}$ for 10 min to remove nitrogen vacancy $\left(V_{\mathrm{N}}\right)$ related defects, $(2)$ molecular beam deposition of an ultrathin Al layer of $1 \mathrm{~nm}$ at RT in a molecular beam epitaxy chamber with a very slow deposition rate of $\sim 0.01 \mathrm{~nm} / \mathrm{s}$, (3) annealing of the sample at $700{ }^{\circ} \mathrm{C}$ for $10 \mathrm{~min}$ in ultrahigh vacuum (UHV), and (4) removal of the resultant thin $\mathrm{AlO}_{x}$ layer in $\mathrm{HF}$ solution. Steps (1)-(3) were carried out in situ in an UHV multichamber system without breaking the UHV condition. Then, after step (4), Pd Schottky contacts with a thickness of $75 \mathrm{~nm}$ and a diameter of $500 \mu \mathrm{m}$ were formed by electron-beam evaporation.

All measurements of hydrogen sensing characteristics were carried out in a small vacuum chamber, which was

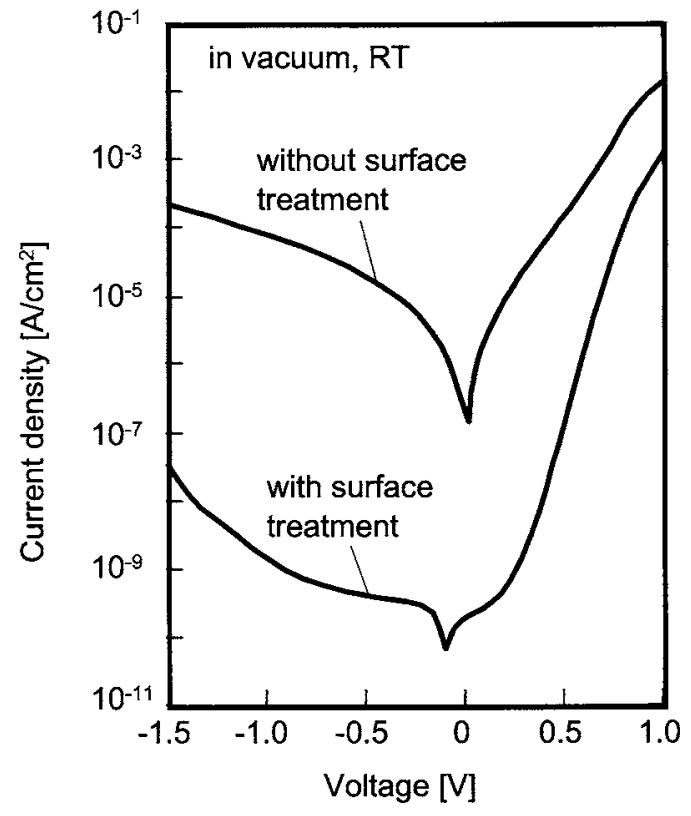

FIG. 2. Effect of the surface control process on $I-V$ characteristics of the diode.

evacuated by a turbo molecular pump. The residual vacuum pressure was $1 \times 10^{-4}$ Torr. High-purity hydrogen gas was supplied into the chamber together with the ambient gas of air $\left(\mathrm{N}_{2}: \mathrm{O}_{2}=7: 3\right)$ or without ambient gas (vacuum). The total pressure was set to be 200 or 750 Torr for air. The partial pressure of hydrogen gas and the background pressure of the chamber were precisely controlled by monitoring the pressure with a vacuum gauge. Repeated pressure transient measurements indicated that the partial pressure transients in the chamber reach the steady-state values within a few seconds for the pressure range used in this study.

$I-V$ and $I-t$ characteristics of the Schottky diode were measured in various ambient gases, using an Agilent 4156A semiconductor parameter analyzer. $C-V$ characteristics were measured by Agilent 4192A LF impedance analyzer.

\section{RESULTS AND DISCUSSION}

\section{A. Effect of surface control process on $I-V$ characteristics of Pd Schottky diode}

The observed effect of the surface control process on the RT current-voltage $(I-V)$ characteristics of the Pd/AlGaN Schottky diode is shown in Fig. 2. Before applying the surface control process, leakage currents in the Pd Schottky diode were very large, about $10^{-3}-10^{-4} \mathrm{~A} / \mathrm{cm}^{2}$ at a reverse bias of $-1.5 \mathrm{~V}$. Current changes caused by hydrogen exposure in such diodes were very small, ${ }^{2}$ one order of magnitude at most at exposure to 10 Torr hydrogen. On the other hand, by applying the surface control process, reverse current densities of the diode were reduced by three to four orders of magnitude down to about $10^{-7} \mathrm{~A} / \mathrm{cm}^{2}$ at a reverse bias of $-1.5 \mathrm{~V}$. Thus, the surface control process is not only effective for Au/Ni Schottky diodes as reported previously, ${ }^{10}$ but also for Pd Schottky diodes. 


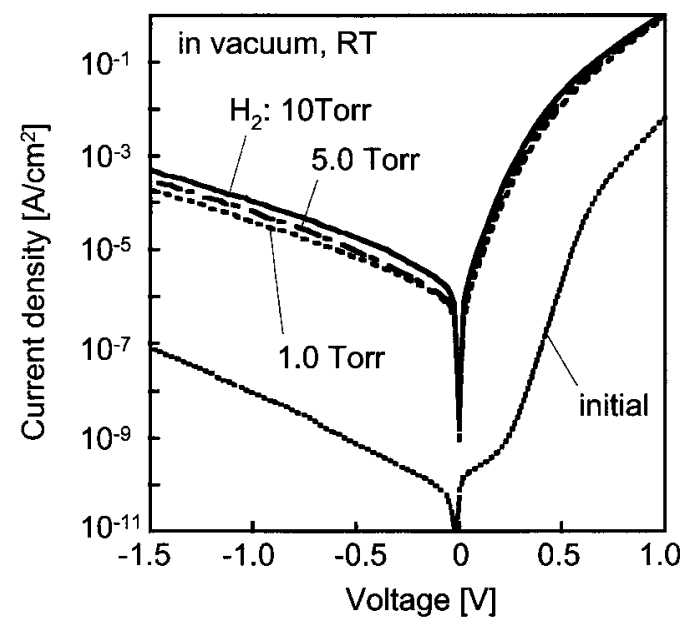

(a)

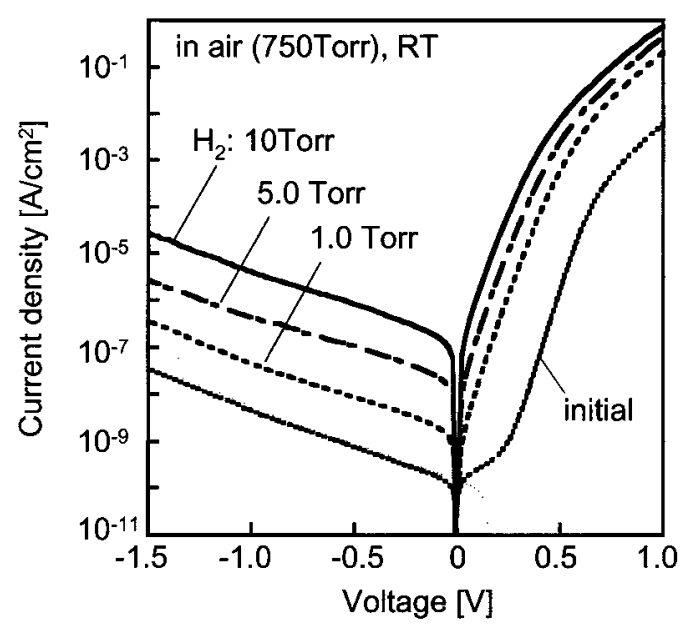

(b)

FIG. 3. Hydrogen-induced changes of $I$ - $V$ characteristics of the diode (a) in vacuum and (b) in air.

This effect not only increased the hydrogen sensitivity of the diode, but also made a detailed analysis of the $I-V$ and $C-V$ characteristics as presented here possible.

\section{B. Steady-state and transient behaviors of diode current after hydrogen exposure}

Hydrogen-induced changes of $I-V$ characteristics of a surface-controlled diode measured at RT in vacuum and in air with a total pressure of 750 Torr are compared in Figs. 3(a) and 3(b), respectively. By introducing hydrogen, large changes in current of three to four orders of magnitude took place in both cases. Thus, the surface control process remarkably enhanced the hydrogen sensitivity of the diode.

However, the pressure dependences of the $I-V$ curves were very different. Namely, in the vacuum case, $I$ - $V$ curves were almost overlapping, showing a very weak dependence on the hydrogen pressure, $P_{\mathrm{H}_{2}}$. On the other hand, the magnitude of hydrogen-induced current change was reduced in air, but the diode showed more systematic dependences of current on the hydrogen partial pressure, $P_{\mathrm{H}_{2}}$.
Turn-on and -off transients of current of the diode measured in vacuum are shown in Figs. 4(a) and 4(b), respectively, for various values of $P_{\mathrm{H}_{2}}$. The observed turn-on behavior was pseudoexponential when the logarithm of current is plotted versus time. Rapid transients with response times $(10 \%-90 \%)$ of several tens of seconds were seen with a weak dependence of response heights on $P_{\mathrm{H}_{2}}$, consistent with the $I-V$ data shown in Fig. 3(a). On the other hand, turn-off transients were extremely slow, as seen in Fig. 4(b). It was also found that turn-off transients could be sped up by introducing air into the chamber, as also shown in Fig. 4(b). Because of this slow turn-off behavior, the steady-state $I-V$ data in Fig. 3(a) were actually taken by repeating a cycle consisting of steps of evacuation, hydrogen introduction, hydrogen removal, and air introduction.

On the other hand, on and off transients of current measured in air are shown in Fig. 4(c). Here, both turn-on and turn-off transients are fairly rapid with response times $(10 \%-$ $90 \%$ ) of several tens of seconds, although turn-off transients seem to be somewhat slower, involving an initial slow falloff transient whose length becomes longer at larger values of $P_{\mathrm{H}_{2}}$. Additionally, the response height shows a systematic dependence on $P_{\mathrm{H}_{2}}$. Again, observed transients were pseudoexponential when the logarithm of current is plotted versus time.

Hydrogen-induced steady-state $I-V$ characteristic changes and current transients observed in this study on Pd/AlGaN Schottky diodes are qualitatively very similar to hydrogen responses of $\mathrm{Pt} / \mathrm{InP}$ Schottky diodes which were recently investigated by our group ${ }^{11}$ where diode behavior was found to be very different in vacuum and in air.

\section{Sensing mechanism and behavior of diode capacitance}

A possible model to explain the observed hydrogeninduced vertical shifts of forward and reverse $I-V$ characteristics is obviously hydrogen-induced reduction of the SBH of the diode. Since Pd possesses highly catalytic properties, and is known to produce atomic hydrogen $\left(\mathrm{H}_{a}\right)$ from hydrogen molecules, SBH lowering is most likely due to the formation of interface dipole by adsorption of atomic hydrogen at the $\mathrm{Pd} / \mathrm{AlGaN}$ interface.

More specifically, such a model tacitly includes the following processes.

1. Hydrogen molecules dissociate on the Pd surface, forming atomic hydrogen.

2. In air, a reaction between atomic hydrogen and oxygen will take place.

3. Atomic hydrogen diffuses through the Pd layer, and reaches the Schottky interface, establishing an equilibrium atomic hydrogen concentration throughout the whole Pd layer.

4. At the Schottky interface, atomic hydrogen is adsorbed at the Schottky interface, and establishes an adsorptiondesorption equilibrium.

5. Positive charge due to adsorbed atomic hydrogen leads to 


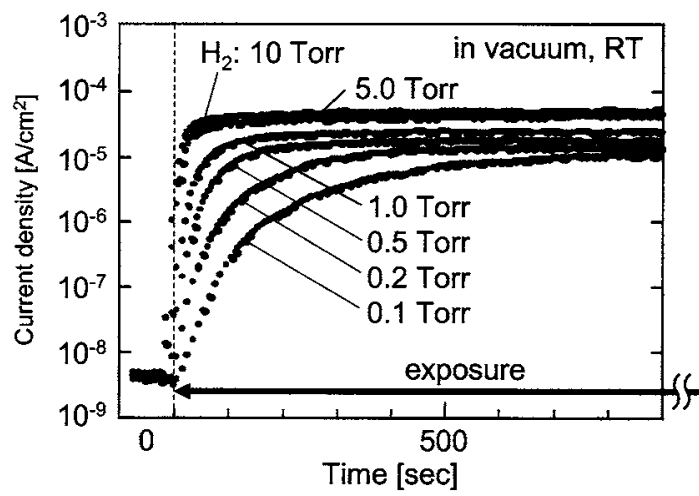

(a)

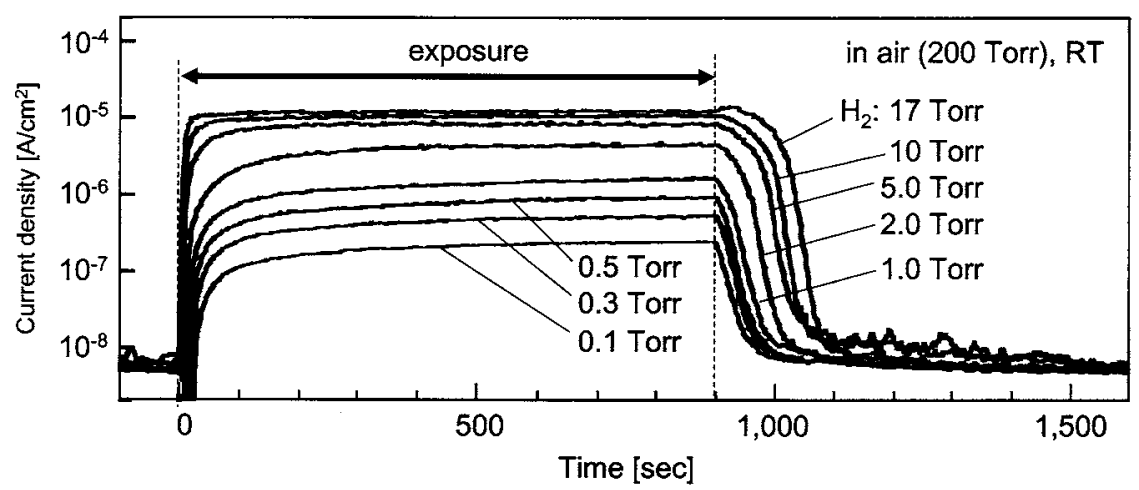

(c)
Fig. 4. Measured transient wave forms of current in the form of $\log I$ vs $t$ plot: (a) turn-on transients in vacuum, (b) turn-off transient in vacuum, and (c) on and off transients in air. formation of dipole at the interface, and reduces the value of $\mathrm{SBH}$.

6. Due to $\mathrm{SBH}$ reduction, both forward and reverse currents increase.

Such an interface dipole model is not an original one here, but dates back to a Si metal-oxide-semiconductor field effect transistor (MOSFET) type gas sensor having a Pd gate discussed by Lundström et al. ${ }^{12}$ a long time ago. However, here, the device structure, the metal, and the semiconductor material are different. The detection mode is also different. Namely, the reverse current change in the $\mathrm{Pd} / \mathrm{AlGaN} / \mathrm{GaN}$ Schottky diode is investigated here, whereas the shift of the threshold voltage of MOSFET was analyzed in the Pd gate $\mathrm{Si}$ MOSFET. Thus, it is worthwhile to investigate the applicability of the above model to the present Pd Schottky diode formed on an AlGaN/GaN 2DEG wafer.

The basic assumption in the above model is that hydrogen exposure changes the $\mathrm{SBH}$ of the diode. In order to confirm this, $C$ - $V$ measurements were performed in vacuum and in air, introducing hydrogen. As an example, steady-state $C-V$ curves at $100 \mathrm{kHz}$ obtained in air are shown in Fig. 5 for various hydrogen pressures. Curves were taken 15 min after the introduction of hydrogen.

Before the introduction of hydrogen, a typical $C$ - $V$ curve for the 2DEG heterostructure capacitor was seen. The flat capacitance toward the positive bias side corresponds to the parallel plate capacitance between the Pd gate and the 2DEG. Upon exposure to hydrogen, parallel shifts of such $C-V$ curve, retaining the same curve shape, were observed. Since
SBH change results in a shift of the threshold voltage of the 2DEG capacitor, the above result directly confirms that introduction of hydrogen causes SBH changes without changing other electronic properties of the system such as doping. Thus, hydrogen passivation of donors does not seem to take place. SBH changes were large, giving a maximum value of about $400 \mathrm{meV}$. A systematic dependence of $C-V$ curve shifts on hydrogen pressure was also seen.

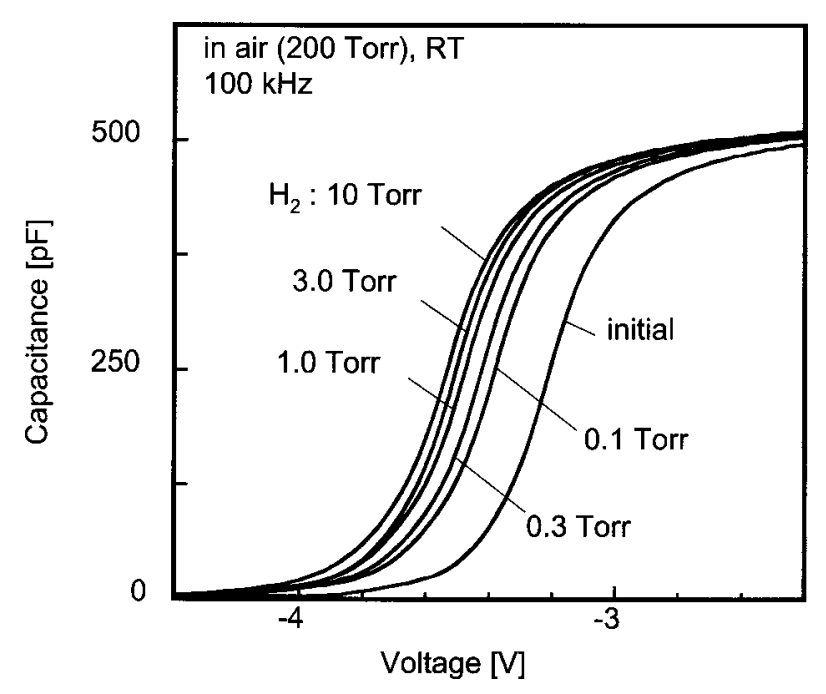

FIG. 5. Measured $C-V$ characteristics of the diode (a) in vacuum and (b) in air. 


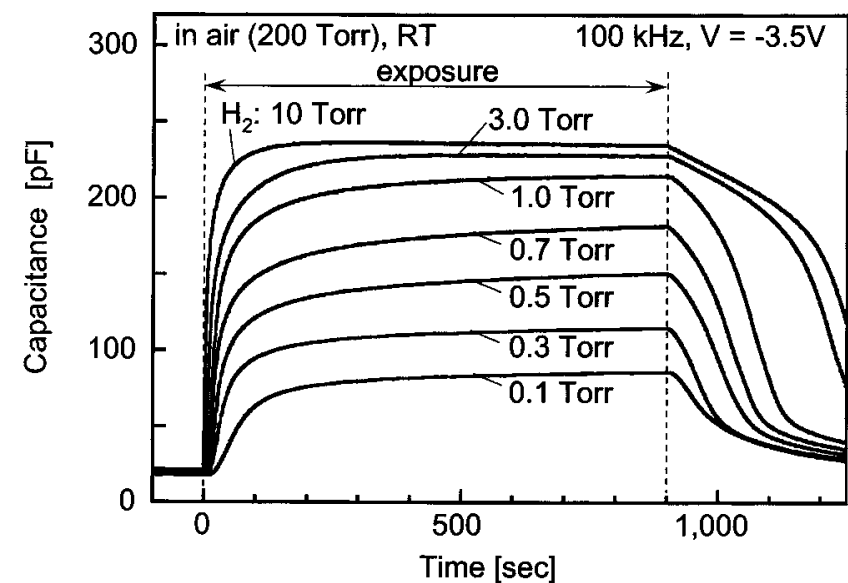

(a)

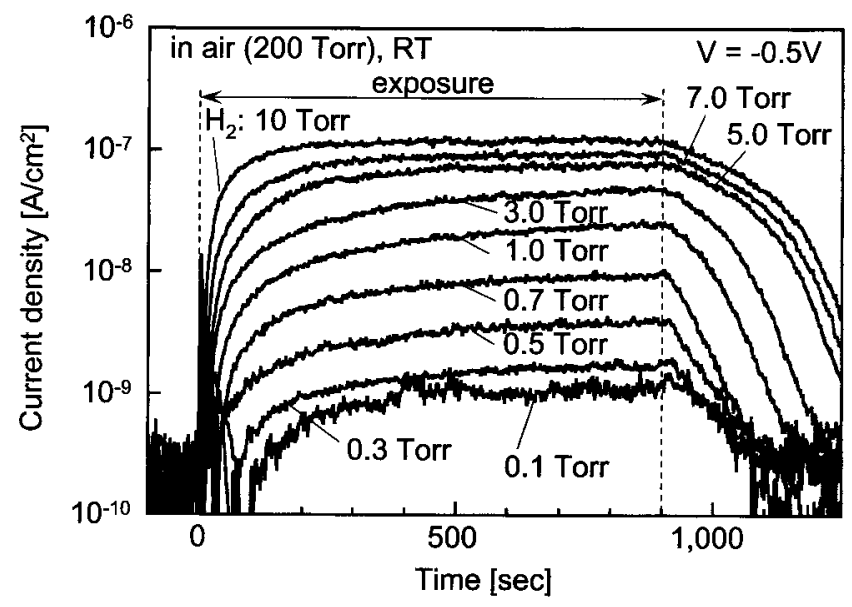

(b)

FIG. 6. (a) Capacitance transients and (b) current transients measured on the same diode in air.

Similar parallel shifts of $C$ - $V$ curves were also seen in vacuum. However, even larger changes of SBH of about $1000 \mathrm{meV}$ were seen in vacuum, with weak dependences of the $C-V$ curve shift on the hydrogen pressure. These features of $C-V$ curves seen in vacuum and in air are well correlated to the behavior of $I-V$ curves shown in Figs. 2(a) and 2(b).

Correlation between the capacitance and current of the diode was more directly seen in transient wave forms. As an example, capacitance transient wave forms and current transient wave forms taken on the same diode placed in air ambient are shown in Figs. 6(a) and 6(b), respectively. It is seen here that capacitance wave forms in a linear scale and current wave forms in a logarithmic scale exhibit strong mutual correlation.

Such a correlation is readily explained theoretically from the above model. Namely, according to the standard thermionic emission (TE) transport model for Schottky barriers, the difference of the logarithm of current before and after SBH change can be related to the decrease of the $\mathrm{SBH}, \Delta \Phi_{B}$, in the following way:

$$
\log I(\infty)-\log I(0)=\frac{q}{k T} \Delta \Phi_{B},
$$

where $I(0)$ and $I(\infty)$ are the steady-state diode currents before and after SBH change, respectively, $q$ is the electronic charge, $k$ is the Boltzmann constant, and $T$ is the absolute temperature. Since the hetero $C$ - $V$ curves in Figs. 5(a) and 5(b) can be roughly approximated by parallel straight lines, capacitance changes can be roughly regarded as being proportional to SBH changes. Thus, change of $\log I$ is roughly proportional to capacitance change, explaining the above correlation. It also indicates that the observed pseudoexponential behavior of currents as plotted in logarithmic scale corresponds to pseudoexponential behavior of SBH change during adsorption of atomic hydrogen.

\section{Quantitative description and analysis of interface dipole formation process}

For further analysis of the experimental data based on the interface dipole model explained above, we need theoretical expressions for interface dipole formation. If we assume that each of the adsorbed atomic hydrogen produces a dipole moment $\mu$ and that the sheet density of such adsorbed hydrogen atom at time $t$ is $N(t)$, the SBH change at time $t$ caused by the dipole layer is given by the following equation:

$$
\Delta \Phi_{B}(t)=-(\mu / \varepsilon) N(t)=-(\mu / \varepsilon) N_{o} \theta(t),
$$

where $\varepsilon$ is the permittivity of the interface, $N_{o}$ is the total number of adsorption sites, and $\theta(t)$ is the "fractional coverage" defined by $\theta(t)=N(t) / N_{o}$.

Combining Eqs. (1) and (2), one obtains the following expression for the normalized transient wave form for the logarithm of the current at time $t, I(t)$.

$$
\frac{\log I(t)-\log I(0)}{\log I(\infty)-\log I(0)}=\frac{\theta(t)}{\theta_{\mathrm{eq}}},
$$

where $\theta_{\mathrm{eq}}=\theta(\infty)$ is the steady-state coverage value. The steady-state current $\log I(\infty)$ is related to the steady-state coverage $\theta_{\text {eq }}$ by

$$
\log I(\infty)-\log I(0)=A \theta_{\mathrm{eq}} \quad \text { with } \quad A=(q / k T)(\mu / \varepsilon) N_{o} .
$$

As for the rate equation which determines the time variation of the coverage, $\theta(t)$, the following two limiting cases can be considered.

\section{Interface adsorption limited case}

This is the case where the adsorption-desorption process mentioned in step (4) becomes the rate-limiting process, where rates of generation and transport of atomic hydrogen are assumed be much quicker so that an equilibrium concentration of atomic hydrogen in the Pd film, whose magnitude depends on the hydrogen pressure, is quickly established. Then, the problem of adsorption and desorption of atomic hydrogen by adsorption sites becomes similar to that of car- 
rier capture and emission processes by a deep level, and the following coverage-linear differential equation is derived:

$$
\frac{d \theta(t)}{d t}=\frac{1-\theta(t)}{\tau_{a}}-\frac{\theta(t)}{\tau_{d}},
$$

where $\tau_{a}$ and $\tau_{d}$ are the effective adsorption and effective desorption time constants, respectively, for the adsorption site for atomic hydrogen at the interface. This gives readily the following exponential form of the solution:

$$
\begin{aligned}
& \theta(t)=q_{\mathrm{eq}}\left(1-\exp \left[-\frac{t}{\tau}\right]\right) \\
& \theta_{\mathrm{eq}}=\frac{1}{1+\left(\tau_{d} / \tau_{a}\right)} \quad \text { and } \quad \tau^{-1}=\tau_{a}^{-1}+\tau_{d}^{-1} .
\end{aligned}
$$

The above equation explains the pseudoexponential behavior of $\log (I(t))$ versus time shown in Figs. 3(a) and 3(b) qualitatively well. The hydrogen pressure dependences of the current response heights and the response speed can be explained through the adsorption time constant, which is expected to be inversely proportional to the concentration of atomic hydrogen in the $\mathrm{Pd}$ film, whose equilibrium value is determined by chemical reactions at the Pd surface. Additionally, the behavior in vacuum can be explained by assuming an infinitely long $\tau_{d}$, giving rise to $P_{\mathrm{H}_{2}}$-independent response heights as well as to extremely slow turn off.

However, actual fitting of experimental data to the exponential forms having the same initial rise-up rate and the final steady-state value gave rather poor results, as shown in Fig. 7(a) for the case of air ambient. Here, Eq. (3) was used as the basis of fitting. This has indicated that the site adsorption/desorption processes at the Schottky interface are not the real rate-limiting processes as assumed above. Additionally, unless one assumes a very unlikely situation of oxygen migration through the Pd layer to the interface, it is also difficult to explain why the same adsorption site gives an infinitely large desorption time constant in vacuum and a finite one in air.

\section{Surface reaction limited case}

The other limit is the surface reaction limited case, where we assume that the interface adsorption process is much faster than the surface reactions to produce atomic hydrogen, so that the coverage of adsorption sites at the inner Schottky interface is directly controlled by the surface reaction. Such situation was previously analyzed by Lundström et al. ${ }^{12}$ and reviewed by Lundström. ${ }^{13}$ According to this, the mostly likely reaction in vacuum producing the atomic hydrogen is the following one:

$$
\mathrm{H}_{2} \underset{d_{1}}{\stackrel{c_{1}}{\rightleftarrows}} 2 \mathrm{H}_{a}
$$

where $\mathrm{H}_{a}$ represents atomic hydrogen, and $c_{1}$ and $d_{1}$ are rate constants for the reaction. This reaction leads to the following rate equation for the fractional coverage:

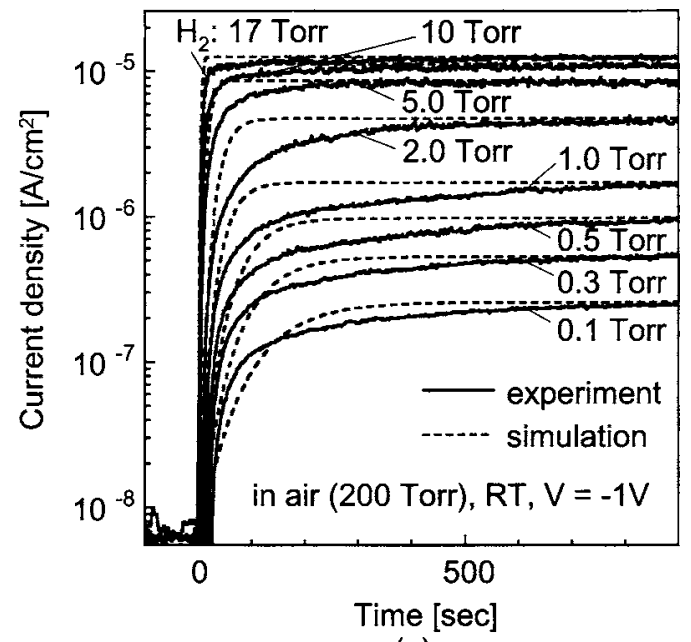

(a)

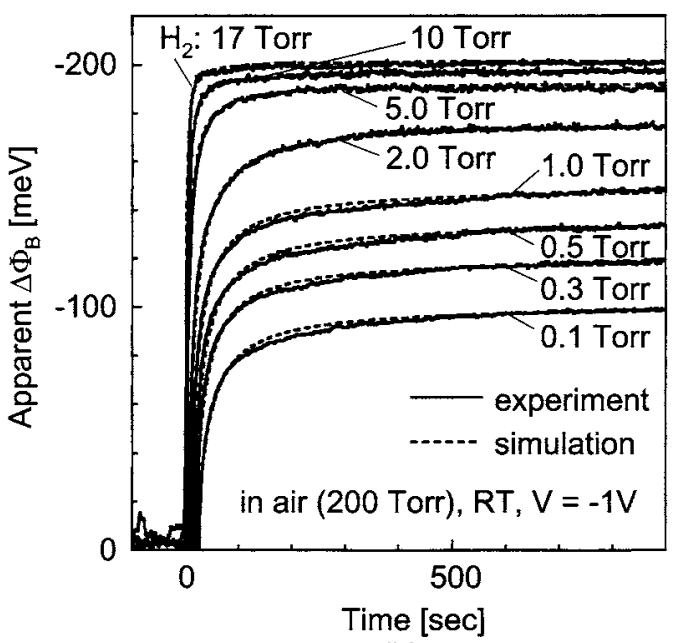

(b)

FIG. 7. Comparison of transient wave forms between theory and experiment for the diode placed in air: (a) exponential fitting and (b) fitting to numerical solutions of Eq. (10).

$$
\frac{\partial \theta(t)}{\partial t}=c_{1} P_{\mathrm{H}_{2}}(1-\theta(t))^{2}-d_{1} \theta(t)^{2} .
$$

This is a coverage-nonlinear equation, and cannot be solved analytically. However, one can determine the steady-state value of the fractional coverage, $\theta_{\mathrm{eq}}$, as follows:

$$
\theta_{\mathrm{eq}}=\frac{\sqrt{\left(c_{1} / d_{1}\right) P_{\mathrm{H}_{2}}}}{1+\sqrt{\left(c_{1} / d_{1}\right) P_{\mathrm{H}_{2}}}} .
$$

This is a Langmuir isotherm type of adsorption behavior for interface coverage by atomic hydrogen.

In the case of the diode placed in air, on the other hand, one has to take account of the reactions between oxygen and atomic hydrogen. Thus, the relevant reactions are the following:

$$
\mathrm{H}_{2} \underset{d_{1}}{\stackrel{c_{1}}{\rightleftarrows}} 2 \mathrm{H}_{a}
$$




$$
\begin{gathered}
\mathrm{O}_{2}+2 \mathrm{H}_{a} \underset{d_{2}}{\stackrel{c_{1}}{\rightleftarrows}} 2 \mathrm{OH}_{a}, \\
\mathrm{OH}_{a}+\mathrm{H}_{a} \underset{d_{3}}{\stackrel{c_{3}}{\rightleftarrows}} \mathrm{H}_{2} \mathrm{O},
\end{gathered}
$$

where $c_{i}$ and $d_{i}(i=1,2$, and 3$)$ are rate constants for the reactions. By ignoring back reactions in Eqs. (9a)-(9c) at room temperature, these reactions give the following rate equation for the fractional coverage, $\theta$ :

$$
\frac{\partial \theta(t)}{\partial t}=c_{1} P_{\mathrm{H}_{2}}(1-\theta(t))^{2}-c_{2} P_{\mathrm{O}_{2}} \theta(t)^{2},
$$

where $P_{\mathrm{O}_{2}}$ is the oxygen partial pressure in air. This is again a coverage-nonlinear equation which cannot be solved analytically. However, the steady-state value of the fractional coverage $\theta_{\text {eq }}$ and the initial rise-up time constant $\tau$ can be obtained as follows:

$$
\begin{aligned}
& \theta_{\mathrm{eq}}=\frac{\sqrt{c_{1} P_{\mathrm{H}_{2}} / 2 c_{2} P_{\mathrm{O}_{2}}}}{1+\sqrt{c_{1} P_{\mathrm{H}_{2}} / 2 c_{2} P_{\mathrm{O}_{2}}}}, \\
& \tau=\frac{\theta_{0}}{\left.(d / d t) \theta\right|_{\theta=0}}=\frac{\sqrt{1 / 2 c_{1} c_{2} P_{\mathrm{H}_{2}} P_{\mathrm{O}_{2}}}}{1+\sqrt{c_{1} P_{\mathrm{H}_{2}} / 2 c_{2} P_{\mathrm{O}_{2}}}} .
\end{aligned}
$$

Now, let us discuss how well the observed behavior of the current transients can be explained by the above equations. First, attempts were made to solve nonlinear differential equations given in Eqs. (7) and (11) numerically on a computer. It was found that the observed current behavior is well reproduced on a computer in both cases in vacuum and in air, by assuming suitable values for reaction parameters. As an example, the results of fitting for the case of air ambient is shown in Fig. 7(b). Here, excellent fitting is seen as compared with the exponential fitting given in Fig. 7(a). Thus, the reaction limited case is the more likely case, both in vacuum and in air.

At room temperature, the rate of the back reaction in Eqs. (6) and (9a) is most probably very slow with a very small value of $d_{1}$ as compared with that of $c_{1}$. In the limit of $d_{1}$ $=0$, Eq. (8) gives $\theta_{\text {eq }}=1$ for the diode in vacuum, independent of the hydrogen pressure. After switching off hydrogen, atomic hydrogen will remain stored in the $\mathrm{Pd}$ bulk region, and no recovery will take place due to the absence of back reaction. These explain the main features of the current transients observed in vacuum. Actually, there is a small dependence of $I_{0 \text { sat }}$ on $P_{\mathrm{H}_{2}}$, most likely due to the nonzero value of $d_{1}$. Additionally, the current shows a small partial recovery after switching off, as seen in the measured data given in Fig. 4(b). At present, the mechanism causing this partial recovery is not clear, and a further study is needed here.

For the behavior in air, the measured steady-state values of the current, $I(\infty)$, are plotted in Fig. 8(a) as a function of the square root of $P_{\mathrm{H}_{2}}$ together with best fitted theoretical curves calculated using Eq. (3b). It has been found that experimental data are well fitted to theory for the value of

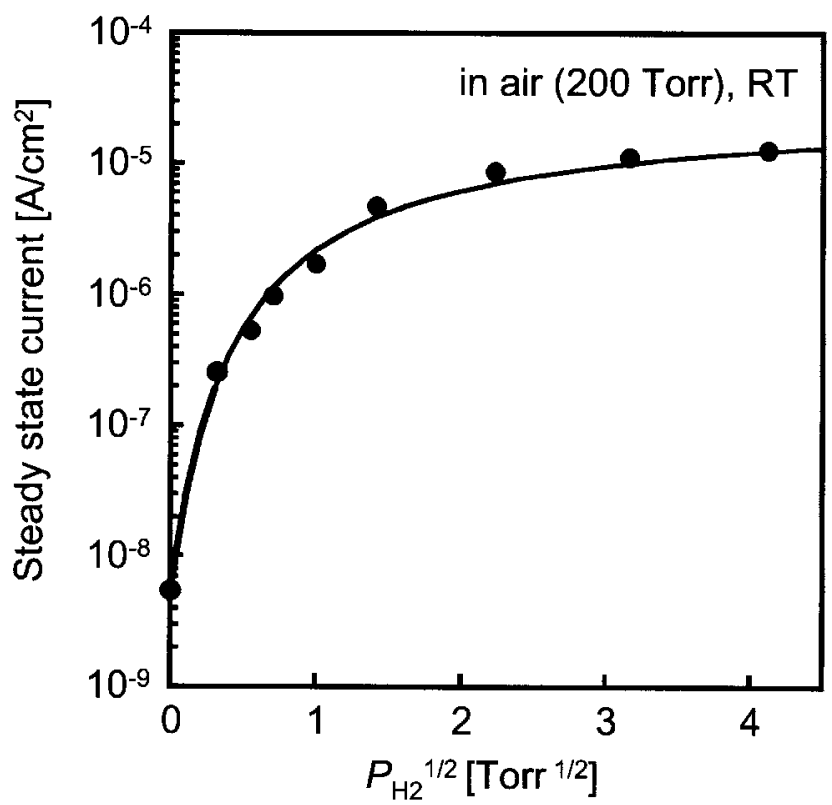

(a)

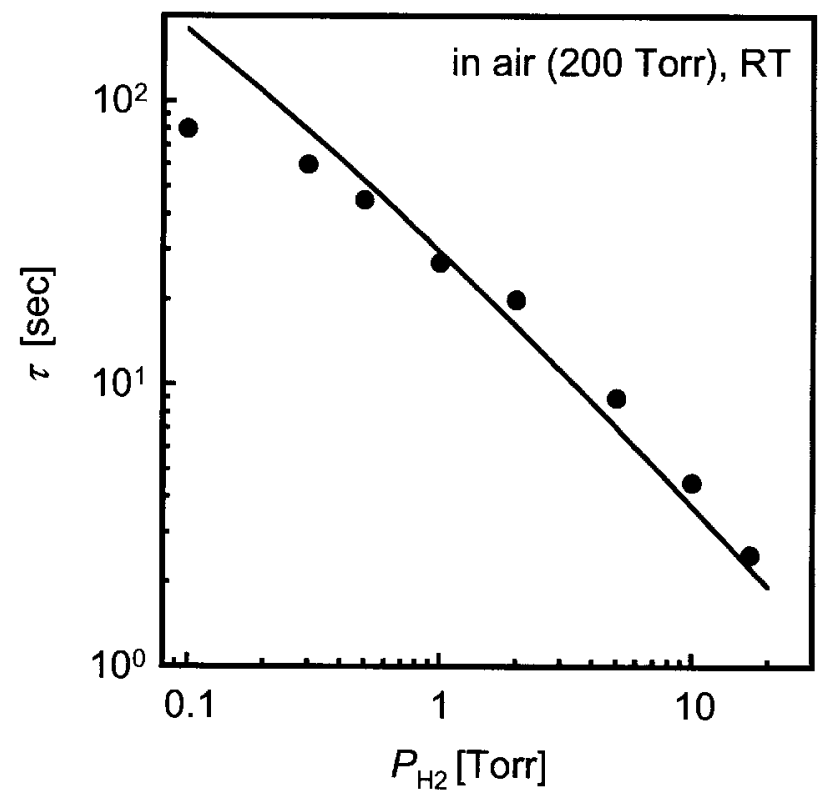

(b)

FIG. 8. (a) Experimental data and theoretical curve for the steady-state current plotted vs square root of hydrogen pressure for a diode place in air, and (b) experimental data and theoretical curve for initial rise-up constant for the same diode.

$\left(2 c_{1} / c_{2}\right)^{1 / 2}=0.065$. In Fig. $8(\mathrm{~b})$, measured values of the initial rise-up time constant $\tau$ are compared with the theoretical curve calculated using Eq. (11b) and $\left(2 c_{1} / c_{2}\right)^{1 / 2}=0.065$. A fairly good agreement is also seen here.

\section{E. SBH change and current transport mechanism}

The values of hydrogen-induced $\mathrm{SBH}$ change, $\Delta \Phi_{B, I-V}$, calculated from current change using Eq. (2), are plotted in Fig. 9 for the diode in air as a function of the square root of hydrogen partial pressure. For comparison, the values of 


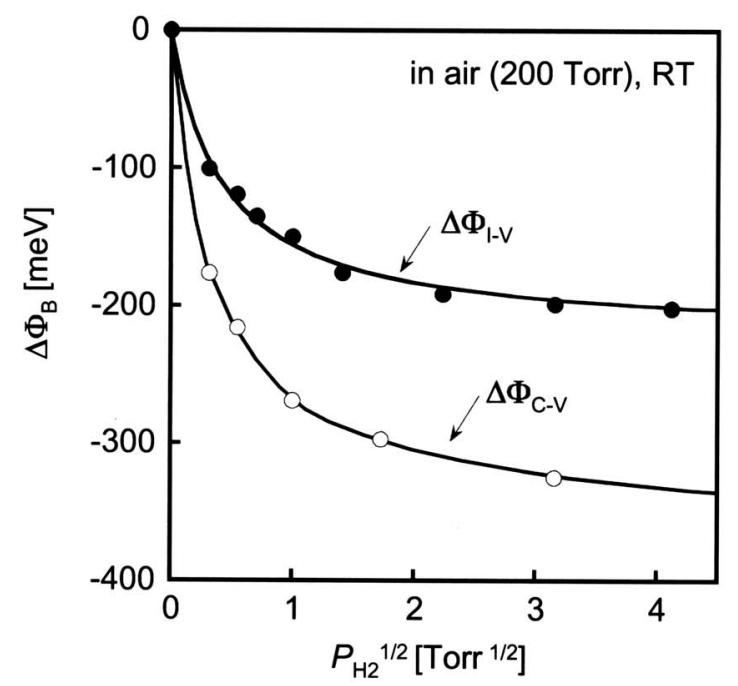

(a)

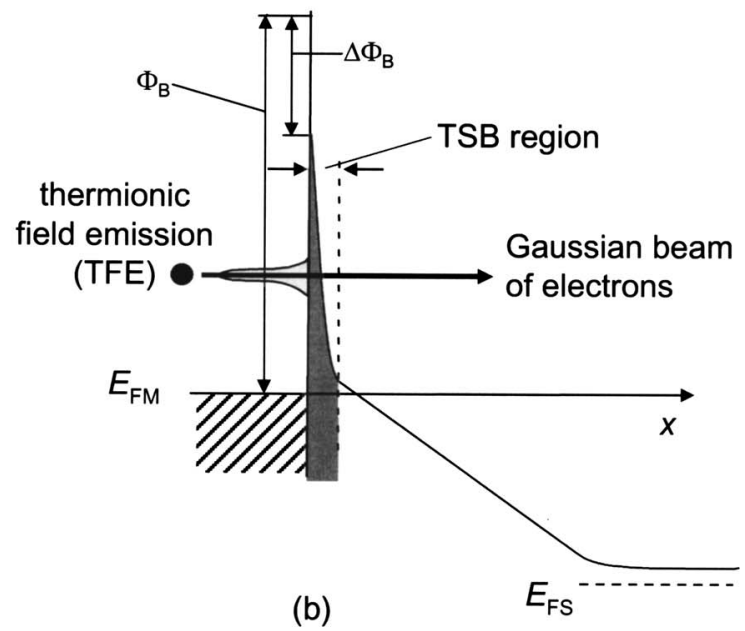

FIG. 9. (a) Values of SBH changes obtained by current measurements and capacitance measurements, and (b) schematic illustration of the thin surface barrier (TSB) model.

hydrogen-induced $\mathrm{SBH}$ change, $\Delta \Phi_{B, C-V}$, which were directly determined by the shift of $C$ - $V$ curves, are also shown in Fig. 9. It is clearly seen that both data do not agree. In fact, the value of the ratio of two barrier heights, $n$ $=\Delta \Phi_{B, C-V} / \Delta \Phi_{B, I-V}$, takes a nearly constant value of $n=1.7$.

This large apparent discrepancy can be explained by the fact that the current transport in $\mathrm{AlGaN}$ and GaN Schottky diodes is not by TE transport, but by the thermionic field emission (TFE) process through a TSB region in accordance with the TSB model. ${ }^{8}$ The physical situation is schematically shown in Fig. 9(b), where the TSB region is produced by nonintentional surface donors such as nitrogen vacancies and oxygen atoms. According to this model, the reverse leakage current flow in the form of a Gaussian beam by tunneling and is expected to become much less sensitive against SBH change due to tunneling. Another strong evidence for TFE transport is the linear relations between the logarithm of the reverse current and the reverse voltage clearly seen in Figs.
2 (a) and 2(b), giving rise to a "reverse ideality factor," $n_{R}$ of about 10 . Such a behavior cannot be explained at all by the TE transport model.

Using the idea of the TSB model, approximate expressions for forward current $I_{F}$ and reverse current $I_{R}$ are given by the following formulas according to an analytical treatment of a square distribution of nonintentional donors with density $N_{\text {DS }}$ on top of a uniform intentional donor distribution with a density $N_{D}:^{8}$

$$
\begin{aligned}
& I_{F}=B \exp \left(-q \phi_{B} / n_{F} k T\right) \exp \left(-q V / n_{F} k T\right), \\
& I_{R}=C \exp \left(-q \phi_{B} / n_{F} k T\right) \exp \left(-q V / n_{R} k T\right),
\end{aligned}
$$

where $n_{F}$ is the "forward ideality factor" given by

$$
n_{F}=\frac{E_{00}}{k T} \operatorname{coth}\left(\frac{E_{00}}{k T}\right) \quad \text { with } E_{00}=E_{00}\left(N_{\mathrm{DS}}\right)=\frac{\hbar}{2} \sqrt{\frac{N_{\mathrm{DS}}}{m^{*} \varepsilon_{S} \varepsilon_{0}}}
$$

and $n_{R}$ is the reverse ideality factor given by

$$
n_{R}=\frac{N_{\mathrm{DS}}}{N_{D}} \frac{1}{1-n_{F}^{-1}} \text {. }
$$

Here, weakly varying preexponential factors were replaced by constants $B$ and $C$ for simplicity.

The above formulas predict exponential dependences of diode currents on both forward and reverse directions in agreement with the measured characteristics shown in Figs. 2(a) and 2(b). Furthermore, the barrier height dependence of current is not by a factor of $\exp \left(q \phi_{B} / k T\right)$ as in the TE transport, but by a factor of $\exp \left(q \phi_{B} / n_{F} k T\right)$ in both forward and reverse directions, causing parallel upward shifts of $\log I-V$ curves in case of SBH reduction. This feature is also apparent in the measured $I-V$ characteristics. The experimental data in Fig. 2(b) give a value of $n_{F}$ of about 1.6 for $P_{\mathrm{H}_{2}}$ $=1$ Torr, and this is in excellent agreement with the above mentioned observed value of $n=1.7$. The observed value of $n_{R}$ of about 10 can be explained by a value of $N_{\mathrm{DS}} / N_{D}$ of about 4 , and this is a reasonable value for the diode subjected to oxygen gettering. Thus, all the features indicate that the observed discrepancy in SBH values is due to the special TFE current transport in the nitride-based Schottky barriers.

\section{CONCLUSION}

This article investigated hydrogen sensing characteristics and mechanism of Pd Schottky diodes that were formed on AlGaN/GaN 2DEG wafer and subjected to a surface control process for oxygen gettering. By applying a surface control for oxygen gettering, leakage currents in $\mathrm{Pd} / \mathrm{AlGaN} / \mathrm{GaN}$ Schottky diode were greatly reduced. Such diodes showed high detection sensitivities and fast turn-on and -off characteristics in air, whereas fast turn-on and very slow turn-off characteristics were seen in vacuum. The sensing dynamics and mechanism can be explained in terms of Schottky barrier height reduction due to the hydrogen-induced interface dipole. Here, dipole formation is controlled by the Langmuir isotherm of adsorption behavior, taking account of oxygen 
reaction. Observed large discrepancies of SBH values have indicated that current transport is due to the thermionic field emission through the TSB region in accordance with the TSB model.

Although the present study has directly confirmed hydrogen-induced SBH change, and has developed good description of steady-state and transient behaviors of the diode based on an interface dipole model, the actual microscopic origin and structure of the dipole is not clear at present. Obviously, it is related to the much disputed problem of the Fermi level pinning at the metal-semiconductor $(M-S)$ interface. Lundström, in fact, argued that Pd modified metal work function and changes the threshold voltage of MOSFET. ${ }^{13}$ However, the dipole picture seems to be more consistent with the present understanding of the $M-S$ interface. These dipoles may be due to hydrogen atoms simply adsorbed on the inner surface of the metal without bonding relation with the semiconductor, or they may be those produced by hydrogen atoms trapped on particular sites within the semiconductor. Otherwise, hydrogen atoms may be modifying or passivating interface states that are responsible for Fermi level pinning. Further work is necessary to clarify this important question.

\section{ACKNOWLEDGEMENT}

This work is supported in part by Grants-in-Aid for Scientific Research [Basic Research (B) No. 18360002: Head
Investigator H. Hasegawa and Exploratory Research No. 18656089: Head Investigator H. Hasegawa] from MEXT, Japan.

${ }^{1}$ http://www.intel.co.jp/research/exploratory/wireless_sensors.htm

${ }^{2}$ K. Matsuo, T. Hashizume, and H. Hasegawa, Appl. Surf. Sci. 244, 273 (2005).

${ }^{3}$ B. P. Luther, S. D. Wolter, and S. E. Mohnley, Sens. Actuators B 56, 164 (1999).

${ }^{4}$ J. Schalwig, G. Muller, U. Karrer, M. Eickhoff, O. Ambacher, M. Stutzman, L. Gorgens, and D. Dollinger, Appl. Phys. Lett. 80, 1222 (2002).

${ }^{5}$ J. Kim, F. Ren, B. P. Gila, C. R. Abernathy, and S. J. Pearton, Appl. Phys. Lett. 82, 739 (2003).

${ }^{6}$ J. Kim, B. P. Gila, G. Y. Chung, C. R. Abernathy, S. J. Pearton, and F. Ren, Solid-State Electron. 47, 1067 (2003).

${ }^{7}$ O. Weideman, M. Herman, G. Steinhoff, H. Wingrant, L. Spetz, M. Stuzmann, and M. Eikhoff, Appl. Phys. Lett. 83, 773 (2003).

${ }^{8}$ H. Hasegawa and S. Oyama, J. Vac. Sci. Technol. B 20, 1647 (2002).

${ }^{9}$ J. Kotani, T. Hashizume, and H. Hasegawa, J. Vac. Sci. Technol. B 22, 2179 (2004).

${ }^{10}$ J. Kotani, M. Kaneko, H. Hasegawa, and T. Hashizume, J. Vac. Sci. Technol. B 24, 2148 (2006).

${ }^{11}$ T. Kimura, H. Hasegawa, T. Sato, and T. Hashizume, Jpn. J. Appl. Phys., Part 1 45, 3414 (2006).

${ }^{12}$ K. I. Lundström, M. S. Shivaraman, and C. M. Svensson, J. Appl. Phys. 46, 3876 (1975).

${ }^{13}$ K. I. Lundström, Sens. Actuators 1, 403 (1981). 